\title{
Acupuncture in the restoration of vasomotor tonus of equine athletes with back pain
}

\begin{abstract}
Injuries along the spinal column of horses are often cited. Acupuncture has been highlighted as a therapy capable of relieving thoracolumbar pain through direct action on vasomotor tone, with a consequent anti-inflammatory and analgesic effect. Therefore, the purpose of this study was to verify the acupuncture action through thermography in vasomotor disorders of the thoracolumbar spine of horses in training. Twenty-six sport horses, with alterations in the thermogram along the thoracic and lumbar spine, were evaluated at different consecutive acupuncture sessions. The results showed significant differences after one, two, four and ten sessions. Moderate lesions tended to recover more clearly than mild lesions. Regardless of the degree of response and number of sessions, acupuncture demonstrated the ability to re-establish the homeostasis of the evaluated region. Regular acupuncture treatment was shown to decrease the severity of thoracolumbar spine vasomotor injuries and provide better quality of life for horses in training.
\end{abstract}

Keywords: toracolumbar, lesions, diagnosis, horses, athletes, back pain
Volume 7 Issue 4 - 2018

\author{
Camila AO Bello,' Andre RC Barreto Vianna, ${ }^{2}$ \\ Kaique Nogueira,' Anna C Santos,' Eduardo \\ MM Lima' \\ 'Faculty of Agronomy and Veterinary Medicine, University of \\ Brasilia (UnB), Brazil \\ 2Department of Veterinary Medicine, Federal University of \\ Lavras (UFLA), Brazil
}

\begin{abstract}
Correspondence: Eduardo MM Lima, I Faculty of Agronomy and Veterinary Medicine, University of Brasília (UnB), ICC Sul, Darcy Ribeiro University Campus, Cx, Postal 4508, Federal District, Brazil, DF 70910-970, Email limaemm@unb.br
\end{abstract}

Received: March 07, 2018 | Published: July 05, 2018
Abbreviations: $\Delta \mathrm{T}$, temperature difference; $\mathrm{CG}$, control group; $\mathrm{TG}$, treated group; M0-M10, moment after 0 to 10 acupuncture sessions; $\Delta \mathrm{Ti}$, initial mean temperature; $\Delta \mathrm{Tc}$, mean temperature of control region

\section{Introduction}

Lesions along the spine and adjacent musculature of horses are very common in the veterinary clinic. The biomechanical requirements required of these structures in the various sports modalities predispose it to acute and chronic disorders. An inflammatory process characterizes some of these disorders, where there is an increase in the local expression of various vasodilating substances, such as interleukins, bradykinins, histamines, peptide $\mathrm{Y}$ and substance $\mathrm{P}$, among others. ${ }^{1} \mathrm{On}$ the contrary, nerve dysfunctions are one of the main causes of chronic back pain in horses. Nerve dysfunctions can increase sympathetic tone, causing local vasoconstriction and triggering neurogenic and myofascial pain. ${ }^{2}$ Acupuncture stands out for its analgesic potential in osteoarticular ${ }^{3,4}$ and neuromuscular alterations.5,6 The use of therapeutic acupuncture for the relief of pain in horses, including pain resulting from thoracolumbar injury has been studied. ${ }^{1,7}$ Zhao et al., ${ }^{8}$ suggest that acupuncture locally regulates the expression of pro-inflammatory mediators, such as substance $\mathrm{P}$, and in this way, the vasomotor action altered by inflammation is restored. Thermographic examination has been widely accepted in the veterinary clinic as an auxiliary, non-invasive method to diagnose spinal injuries in horses. The technique of thermography allows the determination of the surface temperature of the skin. A thermographic finding represented by a hot spot indicates a region where inflammation may be present In contrast, in cases of chronic pain, we can verify cold spots associated with oedema, fibrosis, thrombosis and infarcted tissue. ${ }^{9}$ The thermographic image can provide a complete understanding of vasomotor contro, crucial information for the diagnosis and allow the non-invasive follow-up of the response to the treatment.
For a quantitative thermographic evaluation, the most classical parameter used is the temperature difference or thermal differential $(\Delta \mathrm{T})$ in relation to the contralateral region. For some authors, a difference of $0.5^{\circ} \mathrm{C}$ to $1{ }^{\circ} \mathrm{C}$ of temperature between two corresponding regions is considered acceptable and does not indicate any relevant change. On the other hand, differences greater than this are generally indicative of relevant conditions..$^{9,10}$ It is common for equine athletes to remain in training and competition even though they present with back pain and the training itself can contribute to the establishment of lesions. Therefore, the objective of this study was to verify, through the thermographic technique, the acupuncture action in the vasomotor disorders of the thoracolumbar segment of the spine in equine athletes in training.

\section{Materials and methods}

\section{Experimental design}

We recruited 26 equine athletes, 11 females and 15 males, from equestrian training centres. The equines were between 7 years and 12 years old and had body masses between $450 \mathrm{~kg}$ and 500 $\mathrm{kg}$. All animals were in training, including three horses $(\mathrm{n}=1)$, jumping horses $(n=16)$, polo horses $(n=2)$ and training horses $(n$ $=7$ ). The individuals in the sample presented with alterations in the thermographic examination along the thoracolumbar segment of the spine, including osteoarticular and muscular structures. The healthy horses were separated from inflamed on according to the degree of temperature registered by thermographic analysis of the animals and were separated by the presence of mild, moderate and severe lesions, according to Basile et al., ${ }^{10}$ That is, thermographic differences between -0.5 and $0.9{ }^{\circ} \mathrm{C}(\Delta \mathrm{T})$ were considered normal, thermographic differences between 1.0 and $1.5{ }^{\circ} \mathrm{C}(\Delta \mathrm{T})$ were considered of mild degree, thermographic differences between 1.6 and $2.5{ }^{\circ} \mathrm{C}(\Delta \mathrm{T})$ were considered moderate and greater than $2.5{ }^{\circ} \mathrm{C}(\Delta \mathrm{T})$ severe. A previous selection by history and clinical evaluation was considered. 
All selected animals were free of scars in the region of interest; they were free of topical and systemic medications and of local trichotomy; and they had not been exercised for at least three hours before the thermographic evaluation. All internal body temperatures were within normal limits for equine species, with a mean of $37.05 \pm 0.9^{\circ} \mathrm{C}$. The test was only performed at room temperature, between $19^{\circ} \mathrm{C}$ and 29 ${ }^{\circ} \mathrm{C}$, as suggested by Turner. ${ }^{11}$

Initially, the animals were separated into two groups:

i. Treated group (TG): animals treated with acupuncture weekly (n $=20)$; and

ii. Control group (CG): untreated animals $(n=6)$.

Subsequently, the TG and CG groups were divided into six groups each, according to the moments of evaluation. Thus, the TG group was divided into:

i. M0/TG: it was considered the initial moment before the acupuncture session $(\mathrm{n}=20)$;

ii. M1/TG: the moment after 1 (one) acupuncture session $(n=6)$;

iii. M2/TG: the moment after 2 (two) acupuncture sessions $(\mathrm{n}=13)$;

iv. M4/TG: the moment after 4 (four) acupuncture sessions $(\mathrm{n}=12)$;

v. M5/TG: the moment after 5 (five) acupuncture sessions $(\mathrm{n}=6)$; and

vi. M10/TG: the moment after 10 (ten) acupuncture sessions $(n=8)$.

The control group (without acupuncture treatment) was evaluated at the same moments of the treated group, and was divided into:

i. M0/CG: the initial moment was considered $(n=6)$;

ii. M1/CG: the moment after 1 (one) TG acupuncture session $(\mathrm{n}=6)$;

iii. M2/CG: the moment after 2 (two) sessions of acupuncture of the TG $(n=6)$;

iv. M4/CG: the moment after 4 (four) sessions of acupuncture of the TG $(\mathrm{n}=6)$;

v. M5/CG: the moment after 5 (five) sessions of acupuncture of the TG $(\mathrm{n}=6)$; and

vi. M10/CG: the moment after 10 (ten) sessions of acupuncture of the TG $(n=6)$.

The experiment was developed without inferring with the management of the animals. In this way, the animals continued their usual training, including competitive events. The work was approved by the Ethics Committee on Animal Use (CEUA) of the Institute of Biological Sciences of the University of Brasília (protocol 160100/2013)

\section{Step I - Initial thermographic evaluation}

For the thermographic evaluations, the animals were kept in their own bays, exposed to normal conditions of rest and protected from sunlight or rain. The thermographic images were obtained with the thermographic apparatus (Flir ${ }^{\circledR}$ model E49001 / E40) positioned at a vertical distance of one and a half metres from the dorsal plane of the animals. All evaluations were performed in the same position. To obtain the data, the point temperature of the pathological focus, which was previously diagnosed, was measured three times, obtaining the initial mean temperature $(\Delta \mathrm{Ti})$ (Figure 1A). At the same time, the measurement of the mean temperature of the contralateral region and lesion-free region was performed. The mean temperature of this region $(\Delta \mathrm{Tc})$ was used as self-control (Figure 1B). This methodology was repeated until the tenth session, as shown in Figure 1C. In this way, we neutralized the influence of the variation of the ambient temperature in the evaluated moments, since the possible effects of the external environment were equal for both foci. After obtaining these data, the thermal differential $(\Delta \mathrm{T}: \Delta \mathrm{Ti}-\Delta \mathrm{Tc})$ was calculated, i.e., the temperature difference between the mean of the lesion focus $(\Delta \mathrm{Ti})$ and the mean of the self-control focus $(\Delta \mathrm{Tc})$. The $\Delta \mathrm{T}$ was considered the final data to be evaluated. This methodology was similar to that performed by Um et al. ${ }^{12}$

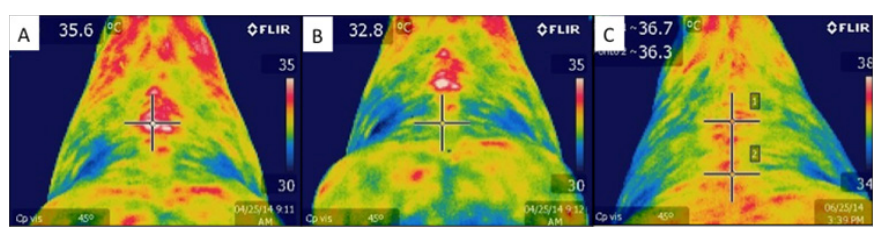

Figure I Thermographic images of the thoracolumbar spine of a training horse at baseline (M0) and after ten sessions of acupuncture (MI0).

(A) Thermographic image at baseline with intersection indicating temperature of $35.6{ }^{\circ} \mathrm{C}$, suggestive of inflammation;

(B) Thermographic image at baseline with intersection indicating temperature of $32.8^{\circ} \mathrm{C}$, suggestive of inflammation;

(C) Thermographic image with intersections indicating the temperature suggestive of inflammation (point I) and the control focus (point 2), after I0 acupuncture sessions

\section{Step II - acupuncture treatment}

The weekly treatment with acupuncture was started after stage I. The thermographic evaluations followed the same methodology mentioned above and were performed once a week, for a total of six evaluations (M0, M1, M2, M4, M5 and M10). The selection of acupuncture points was based on the technique Lun Huan Chiao Ti F $\mathrm{a},{ }^{13}$ and the acupuncture needles were held at the points for $20 \mathrm{~min}$. This technique, often referred as "surrounding the dragon," involves circling an area using points around the perimeter of the lesion; this includes points on the meridian of the bladder and vessel governor. Other points, considered as master and special points related to spinal injuries were used. These were: B-40, B-60, GV-20, E-36, VB-27, B-23, GV-3, GV-4, B-52 and Hua Tuo Jia Ji (Table 1) and (Figure 2).

\section{Statistical analysis}

At the end of the collection, the results obtained were statistically analysed using Graph Pad Prism ${ }^{\circledR}$ software version 6.0 for Windows (GraphPad Software, San Diego, CA, USA). Initially, the Kolmogorov-Sminorv normality test was applied. The comparisons between the two moments of the control group were: M0xM1, M0xM2, M0xM4, M0xM5, and M0xM10. The one-way ANOVA test and the Holm-Sidak post-test were used to verify the significant difference between the moments of the control group compared to the respective moments of the treated group. A $\mathrm{p} \leq 0.05$ was considered statistically significant for all analyses. 
Table I Name and location of the acupuncture points selected for the study

\begin{tabular}{|c|c|c|}
\hline Chinese name & Alternative name & Localization \\
\hline Ming Men & GV - 4 & In the dorsal midline, in the depression between the spinal processes of the L2 and L3 vertebrae. \\
\hline Yang Guan & GV-3 & In the dorsal midline, in the depression between the spinous processes of the L4 and L5 vertebrae. \\
\hline Pang Chang Shu & B-23 & Between the apical extremities of the transverse processes of the L2-L3 vertebrae. \\
\hline Huang Men & B-52 & At the level below the edge of the spinal process of the L3 vertebra. Cun* lateral to the VG5. \\
\hline Yang Ling & B-40 & $\begin{array}{l}\text { Midpoint of the transverse fold of the popliteal fossa, between the semitendinosus and biceps femoralis } \\
\text { muscles. }\end{array}$ \\
\hline Kun Lun & B-60 & Between the lateral condyle of the tibia and the tuberosity of the heel. \\
\hline Bai Hui & GV-20 & In the depression between the spinal processes of the last lumbar vertebra and the first sacral vertebra. \\
\hline Hou San Li & E-36 & On the lateral dorsal surface of the leg, $3.5 \mathrm{~cm}$ distally to the lateral condyle of the tibia. \\
\hline Dan Tian & VB-27 & 0.5 cun* caudal to the dorsocranial iliac spine, above the iliac wing. \\
\hline Hua Tuo Jia Ji & - & 0.5 to I cun* lateral to the depression of the spine process of the L2-L6 vertebrae. \\
\hline
\end{tabular}

* The cun is a proportional and relative unit of measure used to localize the acupuncture points.

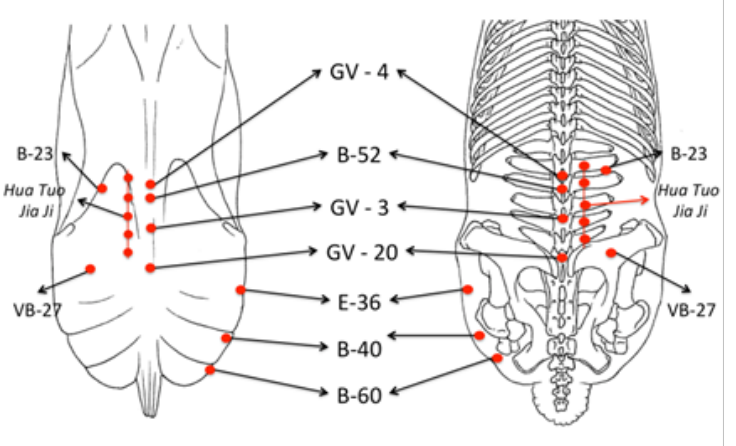

Figure 2 Location of the acupuncture points selected for the study.

\section{Results and discussion}

The thermographic evaluations of the animals of the control group, that is, the animals that were not treated with acupuncture, were performed at the same moments as the acupuncture sessions of the treated groups. We observed that, in these untreated animals, the lesions remained moderate, without presenting a significant difference in temperature (Figure 3) (Figure 4). The results reflected the current hypothesis that acupuncture promotes healing of soft tissue related to the spine of equine athletes. The reduction of $\Delta \mathrm{T}$ after acupuncture treatment was observed at each investigated time point (M1-M10), as expressed in Figure 3 \& Figure 5. There was a reduction of $51.39 \%$ of $\Delta \mathrm{T}$ in $\mathrm{M} 1$ in the group of treated animals, which represented a temperature drop of $0.87^{\circ} \mathrm{C}$ (Figure 5). For the other moments (M2, M4, M5 and M10), there was reduction of $27.27 \%, 66.82 \%, 42.77 \%$ and $62.72 \%$ of $\Delta \mathrm{T}$, respectively. Considering the results, acupuncture exerted a significant influence on the control of the lesions that led to vasomotor abnormalities of the thoracolumbar spine as we can see from the thermographic difference between the control group and the treated group (Figure 3) and (Figure 5). The observed effect was evident from the first session by significantly reducing $\Delta \mathrm{T}$, and it was repeated over two, four and ten sessions. The data obtained in this study reinforced the findings of authors who affirmed the action of acupuncture in the sympathetic nervous system, which acted to promote healing of vasomotor tone and reduce the inflammatory response. . $^{1,2,9}$

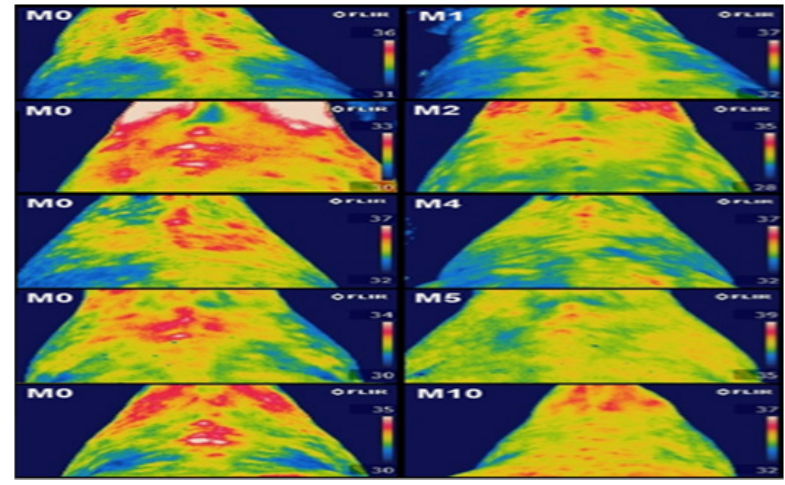

Figure 3 Thermographic image showing the qualitative involution of the injury, demonstrating the effect of acupuncture on vasomotor tone for different time intervals.

The right side scale indicates the maximum and minimum temperatures as a reference to the colours of the thermal map.

$\mathrm{M0}$, Initial time (before any treatment) for each group; $\mathrm{MI}$, after I acupuncture session; M2, after 2 sessions; M4, after 4 sessions; M5, after 5 sessions; MI0, after 10 sessions

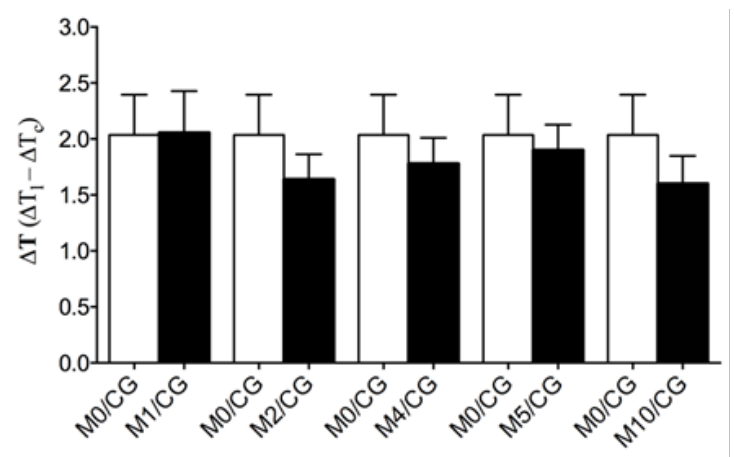

Figure 4 Graph showing average $\Delta \mathrm{T}\left({ }^{\circ} \mathrm{C}\right)$ at different evaluation times compared to $\mathrm{M0}$, temperature reduction $\left({ }^{\circ} \mathrm{C}\right)$ and severity of disorder in the control group (GC).

$M 0$, initial time, before treatment; $M 1$, time after I week; $M 2$, after 2 weeks; M4, after 4 weeks; $M 5$, after 5 weeks; $M I 0$, after 10 weeks; $M_{0}-M$, temperature gradient $\left({ }^{\circ} \mathrm{C}\right)$ between the initial time and after weekly evaluations 


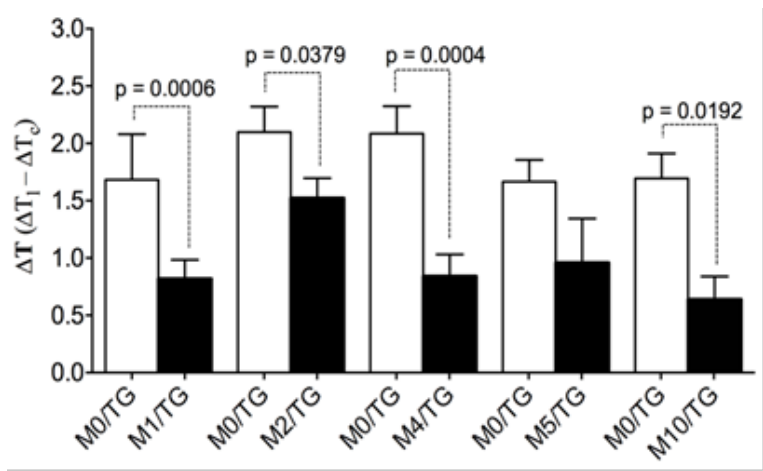

Figure 5 Graph showing $\Delta \mathrm{T}\left({ }^{\circ} \mathrm{C}\right)$ at different evaluation times compared to $\mathrm{M} 0$, temperature reduction $\left({ }^{\circ} \mathrm{C}\right)$, and injury severity degree of the group treated with acupuncture (TG).

M0, initial time before treatment; $M$, time after acupuncture; $M I$, after acupuncture session; M2, after 2 sessions; M4, after 4 sessions; M5, after 5 sessions; $\mathrm{MIO}$, after 10 sessions; $\mathrm{M0}-\mathrm{M}$, temperature gradient $\left({ }^{\circ} \mathrm{C}\right)$ between the initial time and after acupuncture

* Indicates significant difference between times and the absence of $(*)$ means that no statistical difference was found.

Li et al., ${ }^{14}$ and Huang et al., ${ }^{15}$ reported that acupuncture locally stimulated the receptors of complex structures, such as the nerves, blood vessels and lymph nodes. Acupuncture acted by triggering the production of neuropeptides and caused nerve impulses to be sent through the peripheral and autonomic nervous system, thus generating a complete and precise regulation of the chain of cytokines that in turn acted directly on the inflammatory process. ${ }^{16}$ Acupuncture also promoted a decrease in lactate levels and levels of osmotic fragility of erythrocytes in equine athletes and horses that suffered a stressful stimulus. ${ }^{17,18}$ According to the degree of the lesion, except in M2, the final result of the TG showed complete resolution of the inflammatory picture, since the $\Delta \mathrm{T}$ was below $1{ }^{\circ} \mathrm{C}$ and considered physiologically irrelevant. ${ }^{9,10}$ For M2, the effects of acupuncture were sufficient to reduce inflammation from moderate to mild, which also represented an important clinical improvement. In contrast to TG, CG did not show any significant results between any time studied, and neither was observed $\Delta \mathrm{T}$ below $1{ }^{\circ} \mathrm{C}$, which led to the conclusion that the condition was present at all evaluated moments.

In addition, moderate lesions, such as those observed in M1, M2, M4 and M10, presented a more intense response (greater reduction of temperature) to acupuncture than to those of mild (M5) grade. As noted by Parmen et al., ${ }^{19}$ the local acupuncture applied to spinal diseases may decrease local oedema, inflammation, vasodilation or vasoconstriction and the release of histamine or kinins. These signs were stronger in severe and moderate lesions, which may have made it possible to more clearly visualize the effects of local acupuncture repair. On the contrary, the minor injuries were those with chronic characteristics that were caused by the high physical requirements and the functional importance of the spine in the execution of repetitive exercises. The mechanical action of the rider or rider and mount materials, regardless of the modality, acting continuously along the spine should also be considered. According to Schweinitz, ${ }^{2}$ many cases could become incurable as long as the animals remain in athletic life because the cause of the injury will continue to operate. However, given the results obtained, this study suggests that, even in horses with routine training and active participation in competitions, acupuncture has been shown to be clinically effective in restoring vasomotor disorder of the spine, since it was able to reduce the degree of mild injury to absent. Above all, this fact deserves a certain proviso, since no significant difference was observed between the evaluated temperatures.

\section{Conclusion}

The acupuncture treatment exerted an active influence on disorders that lead to vasomotor alteration of the vertebral column of horses submitted to athletic training and competitions. The treatment significantly reduced the abnormal thermal differential immediately after the first acupuncture session and in the second, fourth and tenth sessions. The moderate lesions tended to recover more clearly than mild lesions. However, regardless of the degree of response and number of sessions, acupuncture was clinically able to restore the homeostasis of the region studied at all times in the treated group. The thermographic examination was adequate to detect vasomotor alterations in the thoracolumbar spine and to monitor the response obtained by the treatment with acupuncture. Acupuncture is an adequate therapy to be applied in training horses in the quest to restore or decrease the severity of lesions that lead to vasomotor abnormality in the thoracolumbar spine, thus favouring a better quality of life for these animals.

\section{Acknowledgements}

None

\section{Conflict of Interest}

Author declares there is no conflict of interest.

\section{References}

1. Scognamillo-Szabó MVR, Bechara GH. Acupuncture: scientific bases and applications. Ciência Rural. 2001;31(6):1091-1099.

2. Schweinitz, D Gv. Thermographic evidence for the effectiveness of acupuncture in equine neuromuscular disease. Acupuncture in Medicine. 1998;16(1):14-17.

3. $\mathrm{Xu} \mathrm{L}$, Jing $\mathrm{L}, \mathrm{He} \mathrm{K}$, et al. Treatment of knee osteoarthritis with acupuncture and moxibustion: a randomized controlled trial. Zhongguo Zhen Jiu. 2013;33(10):871-876.

4. Dai Z, Liu HS, Wang SJ, et al. Efficacy evaluation on knee osteoarthritis treated with acupuncture: non-randomized concurrent control trial. Zhongguo Zhen Jiu. 2014;34(4):329-333.

5. Inoue M, Kitakoji H, Yano T, et al. Acupuncture Treatment for Low Back Pain and Lower Limb Symptoms-The Relation between Acupuncture or Electroacupuncture Stimulation and Sciatic Nerve Blood Flow. Evid Based Complement Alternat Med. 2008;5(2):133-143.

6. Yang DL, Zhou WQ, Li J, et al. Comparative study on function and surface electromyograpgy in patients of lumbar disc herniation treated with acupunctrue and moxibustion. Zhongguo Zhen Jiu. 2014;34(4):341-346.

7. Xie H, Asquith RL, Kivipelto J. A review of the use of acupuncture for treatment of equine back pain. Journal of Equine Veterinary Science. 1996;16(7):185-290.

8. Zhao F, Zhu L, Li Y. Therapeutic effects of electroacupuncture (EA) on acute experimental arthritis in rats. Zhen Ci Yan Jiu. 1990;15(3):197-202. 
9. Graf von Schweinitz D. Thermographic diagnostics in equine back pain Vet Clin North Am Equine Pract. 1999;15(1):161-177.

10. Basile RC, Basile MT, Ferraz GC, et al. Equine inflammatory process evaluation using quantitative thermografic methodology. Ars Veterinaria. 2010;26(2):77-81.

11. Turner T. Use of thermography in equine lameness evaluation, North American Veterinary Conference-NAVC. 2007.

12. Um SW, Kim MS, Lim JH, et al. Thermographic evaluation for the efficacy of acupuncture on induced chronic arthritis in the dog. $J$ Vet Med Sci. 2005;67(12):1283-1284.

13. Redfearn T. Surrounding the dragon. Acupuncture in Medicine. 1992;10(2):73-74.

14. Li ZZ, Chen JZ, Guo Y, et al. Influence of acupuncture or thermal acupuncture stimulation at Zusanli (ST 36) on thoracic duct lymph volume and the relevant chemical substances in normal rats/ Zhen ci yan jiu. 2012;37(6):453-457.
15. Huang T, Yang L, Jia S, et al. Capillary blood flow in patients with dysmenorrhea treated with acupuncture. J Tradit Chin Med. 2013;33(6):757-760.

16. Fu X, Zhu ZH, Wang YQ, et al. Regulation of proinflammatory cytokines gene expression by nociceptin/orphanin FQ in the spinal cord and the cultured astrocytes. Neuroscience. 2007;144(1):275-285.

17. Rizzo M, Arfuso F, Giannetto C, et al. Acupuncture needle stimulation on some physiological parameters after road transport and physical exercise in horse. Journal of Equine Veterinary Science. 2017;48:23-30.

18. Rizzo M, Arfuso F, Giannetto C, et al. Cortisol levels and leukocyte population values in transported and exercised horses after acupuncture needle stimulation. Journal of Veterinary Behavior: Clinical Applications and Research. 2017;18:56-61.

19. Parmen V, Pestean C, Ober C, et al. Influence of electroacupuncture on thermal changes in a soft tissue defect. $J$ Acupunct Meridian Stud. 2014;7(5):238-242. 